Kulynych R. L., Rekalov D. G. Forecast and clinical-diagnostic significance of increased expression of beta-2microglobulin in the urine in the process of nephropathy formation in essential hypertension. Journal of Education, Health and Sport. 2020;10(12):65-79. eISSN 2391-8306. DOI http://dx.doi.org/10.12775/JEHS.2020.10.12.007

https://apcz.umk.pl/czasopisma/index.php/JEHS/article/view/JEHS.2020.10.12.007

https://zenodo.org/record/4317779

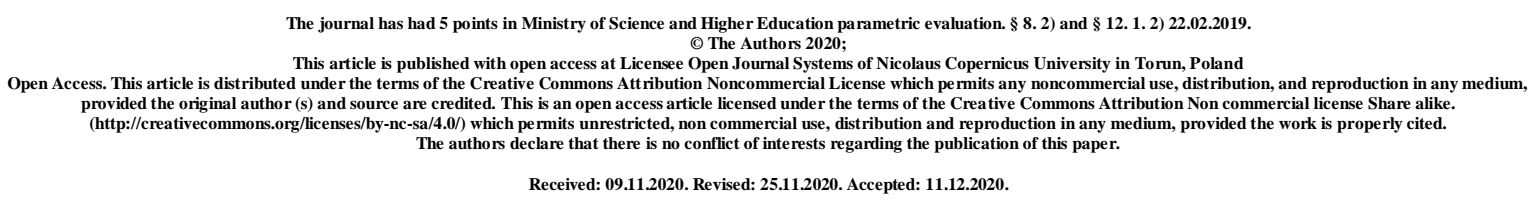

UDC: 616.12-008.331.1-06: 616.61.07

\title{
FORECAST AND CLINICAL-DIAGNOSTIC SIGNIFICANCE OF INCREASED EXPRESSION OF BETA-2MICROGLOBULIN IN THE URINE IN THE PROCESS OF NEPHROPATHY FORMATION IN ESSENTIAL HYPERTENSION
}

\author{
R. L. Kulynych, D. G. Rekalov
}

Zaporozhye State Medical University,

Department of Internal Diseases № 3, Maiakovskyi avenue 26, Zaporizhzhia, Ukraine, 69035 cardiolog1978@gmail.com, ORCID: 0000-0002-8416-0639

\section{Abstract}

Introduction. At present, it is obvious that the prognosis for the life of patients with essential hypertension, according to the cardiovascular continuum, is clearly determined primarily by the involvement in the pathological process of target organs and the severity of their structural and functional changes.

The aim of the study is to identify the clinical-diagnostic and prognostic value of B2MGU as a marker of kidney damage in essential hypertension.

Materials and methods. To achieve this goal, 46 patients with essential hypertension (45.65\% women and $54.35 \%$ men) aged from 28 to 71 years (mean age was $51.88 \pm 2.17$ years), I-II stage of the disease with level of hypertension I-III degree, varying degrees of risk, who did not receive systematic adequate antihypertensive therapy and in which as a result of comprehensive clinical, laboratory and instrumental studies there were no evidence of 
clinically significant chronic kidney disease. The control group consisted of 17 healthy individuals.

Results. In patients with essential hypertension is forming hypertensive nephropathy, which is characterized by statistically significant excessive excretion of beta-2-microglobulin in the urine compared with almost healthy individuals. In patients with increased excretion of beta-2-microglobulin violations of intrarenal hemodynamics were registered significantly more often, violations of functional renal reserve, violations of vascular resistance of renal vessels, in a significantly higher percentage there were pathological changes in circadian rhythm of blood pressure and renal filtration function, the prevalence of concentric and eccentric hypertrophy, proteinuria, which indicates an important clinical, diagnostic and prognostic value of hyper-beta-2-microglobulinuria.

Conclusions. The indicator beta-2-microglobulinuria can be used as an adequate marker of hypertensive nephropathy, as having high sensitivity, specificity, positive and negative prognosis.

Key words: glomerular filtration rate; functional renal reserve; hypertensive nephropathy; urinary albumin excretion; beta-2-microglobulinuria.

Introduction. At present, it is obvious that the prognosis for the life of patients with essential hypertension (EH), according to the cardiovascular continuum, is clearly determined primarily by the involvement in the pathological process of target organs and the severity of their structural and functional changes [1]. Accordingly, the pathogenetic significance of impaired renal function, which is a cause of hypertension and at the same time a manifestation of target organ damage, has been the subject of debate, exacerbated by the presence of a long period of latent renal dysfunction - preclinical stage of renal failure, when routine methods of studying their condition are practically unchanged and do not allow to estimate the severity of functional disorders of the kidneys [2]. Such criteria as proteinuria and increased serum creatinine, usually indicate the late stages of the pathological process in the kidneys, the stage of hypertensive nephroangiosclerosis, which, in turn, contributes to the development of chronic renal failure and indicates a high risk of cardiovascular complications [3].

The main early markers of renal dysfunction are changes in glomerular filtration rate (GFR), increased urinary albumin excretion (UAE) - microalbuminuria (MAU) and decreased functional renal reserve (FRR) [4]. It should be noted that if renal failure is one of the main endpoints of hypertension along with stroke and myocardial infarction, then UAE is an indisputable surrogate point in assessing the effectiveness of antihypertensive therapy [5]. 
A large number of studies have examined the prognostic role of UAE in cardiovascular and renal mortality in diabetes and hypertension. Patients with diabetes mellitus in the presence of UAE have 3-4 times higher cardiovascular mortality and almost 10-fold renal mortality [6]. In patients with EH, UAE has less prognostic value, but is a predictor of kidney damage as a target organ [7]. Therefore, it is especially important for physicians to detect the initial period of renal dysfunction, when pathogenetic treatment can slow down the process of glomerular destruction and fundamentally change the patient's fate, reducing the cardiovascular risk of complications [8]. Moreover, it should be noted that when prescribing treatment and evaluating its effectiveness, it is also necessary to take into account the functional state of the kidneys [9].

Most authors in the pathogenesis of hypertensive nephropathy, in particular its manifestations such as changes in GFR and UAE, play a leading role in capillary-glomerular disorders in relation to which dysfunctions of the tubular apparatus of the nephron and restructuring in the interstitial tissue of the kidneys are secondary [10]. However, there is obvious information that indicates the early involvement of tubules in the pathological process in EH [11]. In 1990 Tomlinson P.A. was one of the first who have been used beta-2microglobulin (beta-2-MG) as a clinical test for tubular dysfunction [12]. The presence of this low molecular weight protein in serum is due to the processes of degradation and repair of individual cell elements, beta-2-MG freely passes through the glomerular membrane during filtration, and then about $99.8 \%$ is reabsorbed in the proximal renal tubules. At disturbances of function of tubules its excretion sharply increases [13]. It should be emphasized that studies about evaluating markers of renal damage in patients with $\mathrm{EH}$ are usually traditionally devoted to such a highly informative indicator as UAE, while data on changes in urinary beta2-MG in this pathology are insufficient, and the obtained data are contradictory [14]. The available literature does not sufficiently reflect the data on the dynamics of beta-2microglobulinuria (B2-MGU) in EH, vaguely formulated the main pathogenetic mechanisms of increased excretion of beta-2-MG in patients with EH, not fully studied the clinical and diagnostic value of this indicator in this categories of patients.

The aim of the study is to identify the clinical-diagnostic and prognostic value of B2MGU as a marker of kidney damage in EH.

Materials and methods. To achieve this goal, 46 patients with EH (45.65\% women and $54.35 \%$ men) aged from 28 to 71 years (mean age was $51.88 \pm 2.17$ years), I-II stage of the disease with level of hypertension I-III degree, varying degrees of risk, who did not receive systematic adequate antihypertensive therapy and in which as a result of 
comprehensive clinical, laboratory and instrumental studies there were no evidence of clinically significant chronic kidney disease. The control group consisted of 17 healthy individuals. All respondents agreed to participate in the study. Daily monitoring of blood pressure (BP) was performed using a device "Cardiotens-01" ("Meditech", Hungary). The monitor was activated according to the standard protocol every 15 minutes during the day (06:00-22:00) and every 30 minutes during sleep (22:00-06:00), the obtained data with the definition of indicators were analyzed using the software of the device. Evaluated daily indicators: ambulatory blood pressure monitoring (ABPM), mean clinic systolic and diastolic BP, systolic BP (SBP) daytime and nighttime (SBPd and SBPn), diastolic BP (DBP) daytime and nighttime (DBPd and DBPn), load indices - area index (AI) and time index (TI) for SBP and DBP. The daily index was studied to determine the type of circadian rhythm of blood pressure (subjects in whom SBP and DBP at night decreased by $10-20 \%$ compared to the daytime period, were classified as "dipper"; less than 10\% - "non-dipper"; with a steady increase in blood pressure at night - "night-peaker" (negative values of the daily index; with a decrease in night blood pressure by more than $20 \%$ - "over-dipper"). Determined the variability of blood pressure separately during the day and night for SBP and DBP, and also calculated the absolute value and rate of morning rise in blood pressure.

Studies of renal hemodynamics were performed in the horizontal position of the patient on the device "Sonoline Versa Plus" (SIEMENS, Germany), using color Doppler mapping and pulsed wave Doppler with a convex sensor. Blood flow in the renal arteries was studied at the level of the main trunk (MRA), segmental (SRA) and interlobular branches of the renal arteries (IRA) in the spectral Doppler mode, assessing the linear indicators of blood flow: peak systolic and diastolic velocity, mean speed. Peripheral vascular resistance (resistance) was also used to characterize renal blood flow: S / D - systolic-diastolic ratio of velocities, PI - pulsation index, RI - resistance index (peripheral resistance). The calculations were performed automatically when processing Doppler curves according to the appropriate formulas. GFR was calculated standard according to the formula Cocroft-Gault, with GFR > $120 \mathrm{ml} / \mathrm{min}$ regarded the condition of the kidneys as hyperfiltration, a level of less than 80 $\mathrm{ml} / \mathrm{min}$ - as a decrease in GFR. Studies of FRR, which is a clinical marker of intraglomerular hypertension, were performed on all subjects, using GFR on endogenous creatinine clearance before and after oral protein loading in conditions of adequate water regime according to the modified method. FRR, the quantitative measure of which is the difference between the stimulating GFR and its basal level, expressed as a percentage of the initial GFR level, was calculated by the formula FRR $=((\mathrm{R} 2-\mathrm{R} 1) / \mathrm{R} 1) * 100 \%$, where R1 is 
the initial GFR value, R2 - marking GFR after the test. The following scale was used to assess FRR: an increase in GFR upon protein stimulation of more than 10\%, compared with basal GFR, was regarded as preserved FRR; an increase in GFR by 5-10\% was interpreted as a decrease in FRR; increase in GFR by less than 5\% or decrease it after stimulation, was defined as depleted FRR. Serum creatinine concentration was determined by a unified method using the Jaffe color reaction (Popper method). The level of urinary albumin and beta-2-MG excretion (morning portion) were examined by enzyme-linked immunosorbent assay on a semi-automatic analyzer "DigiScan" (Austria), using kits from "Orgentec" according to the instructions in the Training Medical and Laboratory Centre of Zaporizhia State Medical University, certified by the Ministry of Health of Ukraine (certificate № 039/14).

Echocardiographic examination was performed on a digital Doppler ultrasound scanner "Ultima Pro-30" (RADMIR, Ukraine) according to a standard protocol. Normalized by body surface area, the value of the indexed indicator (left ventricular mass index, LVMI) was calculated by Devereux R. et al. (normal values of LVMI were considered indicators for men $<125 \mathrm{~g} / \mathrm{m}^{2}$, for women $<110 \mathrm{~g} / \mathrm{m}^{2}$ ), to assess the type of left ventricle (LV) remodeling, the relative wall thickness (RWT) was calculated according to A. Ganau. According to the classical notions of structural and functional changes of the myocardium for the normal geometry of the LV was considered RWT $<0.45$ in normal LVMI, concentric cardiac hypertrophy was diagnosed at RWT > 0.45 and increased LVMI, eccentric LV hypertrophy for RWT < 0.45 and increased LVMI, concentric remodeling - at RWT > 0.45 and normal LVMI.

Distribution normality tests were performed (Kolmogorov-Smirnov (D), Lilliefors and Shapiro-Wilk (W) criteria). Comparison of groups on a qualitative binary basis was performed using the criterion $\chi^{2}$ with the analysis of correlation tables. In the case of a distribution other than normal, or analysis of ordinal variables, the Mann-Whitney U test was used for two unrelated samples, and for a larger number, the Kruskal-Wallis $\mathrm{H}$ test was followed by a Games-Howell comparison. The relationship between quantitative indicators was assessed using correlation (Spearman's ratio) and regression analyzes. Evaluation of the effectiveness of diagnostic studies was performed using qualitative reference data using fourfield tables, using calculated indicators: sensitivity (Se), specificity ( $\mathrm{Sp}$ ), predictability of positive (PVP) and negative (PVN) results. The results of the study are presented in the form of median and interquartile range (25 and 75 percentiles) in parentheses. The results of the study were processed using the statistical software package "SPSS 15", "Microsoft Excel 
2003" and "STATISTICA® for Windows 6.0" (StatSoft Inc.), for all types of analysis were statistically significant differences at $\mathrm{p}$ less than 0.05 .

Results and discussion. According the analysis of the level of excretion of beta-2-MG in the urine of the control group, the following results were obtained: the nature of the distribution of the obtained values of B2-MGU differs from normal (statistics of differences Kolmogorov-Smirnov 0.176 with a probability of Lilliefors $0.171 \mathrm{p}=0.11$ ). B2-MGU values ranged from 7 to $316 \mu \mathrm{g} / \mathrm{L}$ and were in the group of almost healthy individuals $121(22 ; 162)$ $\mu \mathrm{g} / \mathrm{L}$, and in two examined persons the concentration of beta-2-MG in the collected urine analysis were below the range of detectable values for enzyme-linked immunosorbent assay method of determining the urinary concentration of microproteins. We calculated the reference values for the upper limit of B2-MGU for the norm, which corresponds to the 99th percentile of the values of the sample of almost healthy individuals and is about $400 \mu \mathrm{g} / \mathrm{L}$ $(402 \mu \mathrm{g} / \mathrm{L})$. In patients with $\mathrm{EH}$, the level of B2-MGU was $320(118 ; 820) \mu \mathrm{g} / \mathrm{L}$, which is significantly higher than similar indicators of almost healthy individuals by 2.6 times. We divided all patients with EH according to the level of B2-MGU into 2 subgroups: 1 - with normal level (21 patients) and 2 - with excessive excretion (more than the calculated value of $400 \mu \mathrm{g} / \mathrm{L}$ ) excretion of beta-2-MG in the urine -25 people. The obtained subgroups were comparable by sex (female / male ratio: 10/11 and 12/13 for 1st and 2nd groups, respectively), age (1st subgroup $47(46 ; 57.5)$ years and 2 nd $-52(45,5 ; 60.5)$ years), stage of the disease (in both groups the vast majority were patients with stage II og EH) and the degree of hypertension. The analysis of the obtained data revealed that patients of subgroup 2 were characterized by significantly high differences in the indices of vascular resistance of the renal vessels (in patients of this subgroup RI and PI SRA (7.81\% and 10.62\%) and RI and PI IRA (by $9.23 \%$ and $11.54 \%$ ) were higher than in 1st subgroup. Significant differences were also registered in the rate of urinary albumin excretion $(20.31(18.7 ; 21.39) \mathrm{mg} / \mathrm{L}$ and $334(96,64$; 615.06) $\mathrm{mg} / \mathrm{L}$, respectively, and the incidence of proteinuria was significantly higher in $2 \mathrm{nd}$ subgroup $-\chi^{2} 6.83$ at $p=0.009$ ). In terms of GFR (Cocroft-Gault) subgroups did not differ significantly, due to the presence of 2 nd subgroup, both patients with initially reduced GFR and patients with hyperfiltration, which, in general, offset the reduced filtration capacity of the kidneys in this subgroup, but further analysis of the frequency of detection of reduced GFR and hyperfiltration, the data show that in patients with EH with an increase in B2-MGU is significantly more often recorded pathological changes in GFR, mainly a decrease in GFR. At the same time, the analysis of FRR revealed the following patterns: the frequency of detection of impaired FRR (reduced and depleted) in patients of 2nd subgroup was 
significantly higher than in the 1 st $\left(\chi^{2} 5.29, \mathrm{p}=0.021\right)$. Moreover, it should be noted that FRR in patients of 2nd subgroup averaged $2.11(-0.66 ; 5.28) \%$, which is less than 2 times the rate of normal excretion of beta-2-MG in the urine - 1st subgroup $(5.36(3.57 ; 6.78) \%)$. Analysis of the variants of the circadian rhythm of BP according to the subgroups showed that the frequencies of the types "dipper", "non-dipper", "night-peaker" and "over-dipper" in the 1st subgroup was $45.45 \%, 27.27 \%, 0 \%$ and $27.27 \%$, respectively, in the 2nd subgroup, these indicators were $28.57 \%, 45.71 \%, 22.86 \%$ and $2.86 \%$, which indicates a significantly higher prevalence of circadian physiological rhythm disorders in patients with elevated excretion of beta-2-MG and indicates a much greater prerequisite for the defeat of target organs and the development of cardiovascular pathology in patients of this subgroup. Interesting data were obtained during the analysis of changes in the structural and functional rearrangement of the heart in EH depending on the level of B2-MGU: so LVMI in 1st subgroup was 89.36 (80.38; 93.98) $\mathrm{g} / \mathrm{m}^{2}$, which is $67.48 \%$ less than in patients with hyper-B2-MGU - 149.66 (125.96; 168.37) $\mathrm{g} / \mathrm{m}^{2}$ (Fig. 1).

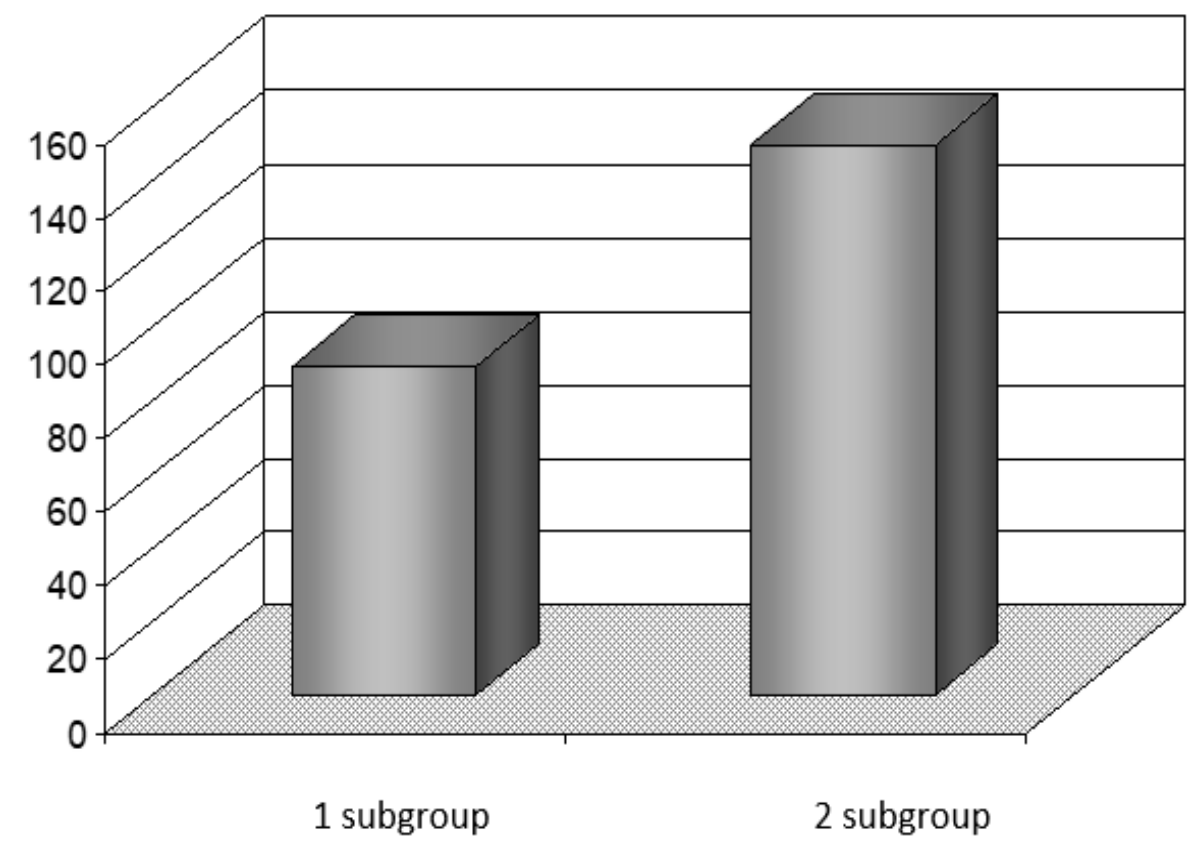

Fig. 1. Indicators of LVMI of the examined patients with hypertension depending on the level of B2-MGU

It should be noted that the prevalence of concentric and eccentric hypertrophy, the most unfavorable options for cardiac remodeling at risk of cardiovascular complications, was 
also significantly more often registered in patients of 2 nd subgroup compared with 1 st $\left(\chi^{2}\right.$ was 9.67 at $\mathrm{p}=0.002)$.

To identify and assess the degree of relationship between the studied indicators and the level of B2-MGU, we conducted a correlation analysis, the results of which allowed us to note a positive correlation between the indices of blood pressure (especially for DBP), on the one hand, and the value of B2-MGU, on the other (for AI-DBPn - B2-MGU R was 0.52, for TI-DBPmean and TI-DBPn 0.47 and 0.5, respectively). The relationship between LVMI and B2-MGU was also directly proportional, with a correlation coefficient of 0.43 for this pair. The relationship between the value of RI and PI SRA and RI and PI IRA and the excretion of beta-2-MG were also significant, but less dense $(\mathrm{R}=+0.29,+0.31$ and +0.34 and $+0,35$, respectively, $\mathrm{p}<0.05$ in all cases), for $\mathrm{S} / \mathrm{D}$ ratio $\mathrm{R}$ was 0.3 . For FRR and emission fraction, in contrast to others, the interdependence with B2-MGU was inversely proportional $(\mathrm{R}=-$ 0.44 and -0.22 , respectively), as the FRR decreased (increased manifestations of intraglomerular hypertension), the level of beta-2-MG significantly increased in urine.

Further, in order to assess the nature of the relationship between indicators that reflect the functional state of the kidneys, and to build a functional relationship between the variables studied, we conducted a regression analysis, during which the level of urinary albumin excretion was taken as an independent argument. adequately reflects the process of kidney damage in patients with EH [15], and as a dependent variable was considered the value of B2MGU. It turned out that the obtained relationship between variables with a sufficient degree of accuracy and adequacy was approximated by a regression model of polynomial nature: albuminuria $(\mathrm{AU})=-43.45+624.57 * \mathrm{~B} 2-\mathrm{MGU}-121.05 * \mathrm{~B} 2-\mathrm{MGU}^{2}$ (Fig. 2). Thus, we demonstrated a statistically significant close relationship between the level of B2-MGU and urinary albumin excretion in patients with $\mathrm{EH}$ and allows the use of B2-MGU as a marker of kidney damage in EH (according to the high value of the coefficient of determination).

To establish the diagnostic value of the method for determining hyperbeta-2-MGU (B2-MGU excretion less than $400 \mu \mathrm{g} / \mathrm{L}$ ) as a marker of hypertensive nephropathy in patients with EH, we determined indicators such as sensitivity, specificity, positive and negative significance of the method. As a reference method, used the level of MAU, a traditional and generally accepted laboratory indicator of impaired renal function. Based on the comparison of the obtained results in the determination of MAU and B2-MGU, the initial data necessary for the calculation of indicators were determined: the number of really positive (number of patients with EH with nephropathy diagnosed by both reference and proposed method), really negative results (number of patients without renal impairment according to both the reference 
and the test method) and the number of false-positive (number of patients with hypertensive nephropathy detected by the method of assessing the level of B2-MGU, in those examined without kidney damage according to MAU) and the number of false-negative results (the number of cases of absence of renal dysfunction according to the method with the assessment of the level of B2-MGU in patients with established hypertensive nephropathy by the reference method).

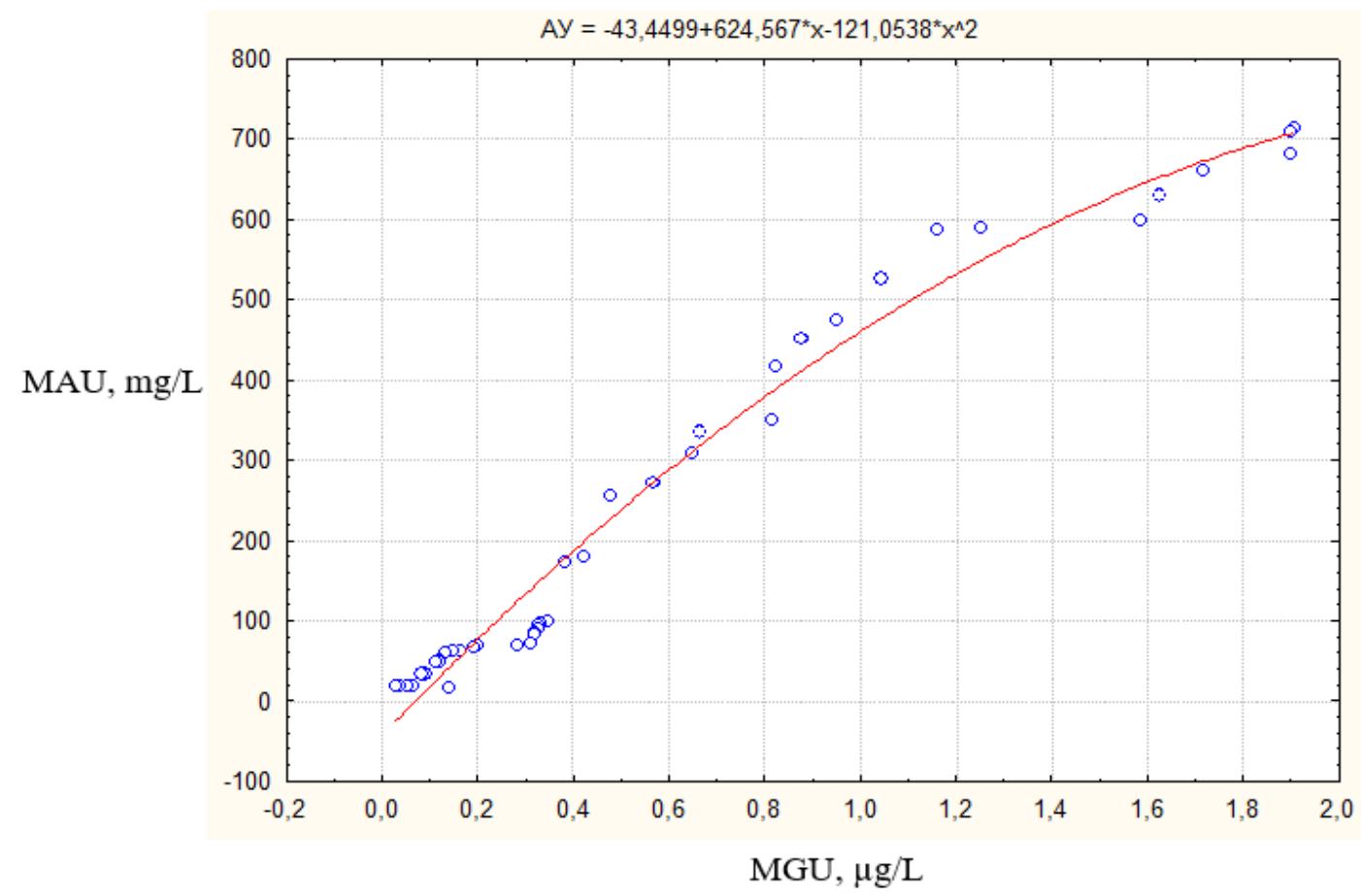

Fig. 2. The results of regression analysis of the relationship between the level of urinary albumin excretion and the value of B2-MGU in patients with EH

According to our data, the method of diagnosing hypertensive nephropathy by the level of B2-MGU excretion in the daily urine (more than $400 \mu \mathrm{g} / \mathrm{L}$ ), has a fairly high sensitivity $(\mathrm{Se}=85 \%)$, specificity $(\mathrm{Sp}=83.33 \%)$ and sufficient positive and negative prognosis $(\mathrm{PVP}=97.14 \%$ and $\mathrm{PVN}=45.45 \%)$ in relation to the diagnosis of nephropathy in EH.

The data obtained by us are consistent with some works of other researchers, in this study there were registered an increase in protein of surface antigens of cell nuclei in patients with $\mathrm{EH}$, and the dynamics of this microprotein, the authors evaluated the effectiveness of therapy on the background of treatment with spirapril [16]. Also there were noted that the determination of low molecular weight urinary proteins (beta2- and alpha1-MG, retinolbinding protein, urinary protein-1) is a valuable clinical tool for assessing functional 
impairment of the kidneys in adults and children [17]. At that time after examination of about 3 thousand patients with $\mathrm{EH}$ with varying degrees of hypertension, there were found in more than $30 \%$ of patients increased levels of urinary B2-MG, explaining this by overdiagnosis of $\mathrm{EH}$ and linking the data with the presence of this group of persons with primary kidney damage and symptomatic hypertension [18]. Researchers found no significant differences in the level of urinary excretion of B2-MG in EH, in contrast to patients with renal parenchymal hypertension, in whom a significant increase in B2-MGU, which allows, according to the authors, to use this indicator as a differential-diagnostic criterion between the above nosologies [19]. Despite this, another group of researchers noted in their work a statistically significant increase in the level of B2-MG excretion in hypertensive patients with low plasma renin activity compared with patients with normoreninemia [20]. Laser nephelometry was used to evaluate the dynamics of uroproteins on the background of therapy with various antihypertensive drugs in patients with mild and moderate hypertension [21]. The analysis of the data obtained by these authors testified to the sufficient informativeness of the B2-MGU indicator, which, together with the excretion of other microproteins, allows not only to assess the functional state of the kidneys, but also to monitor the effectiveness of therapy and clarify the prognosis. The increased level of excretion of B2-MG in the urine in EH in comparison with almost healthy, also were indicated in some works, assessing the dynamics of this indicator depending on the functional state of the kidneys. They concluded that as the reserve capacity of the kidneys to increase GFR after the test with oral protein load, the level of B2MGU increases significantly [22]. Another scientist noted the presence of hyper-beta-2-MGU in patients with $\mathrm{EH}$, without specifying the possible mechanisms of increased excretion of this low molecular weight protein and its relationship with the degree of hypertension and stage of the disease [23]. Increased level of urinary excretion of markers of dysfunction of the tubular epithelium in EH (B2-MG and lysosomal enzyme N-acetyl-beta-D-glucosaminidase) noted in few works, emphasizing that the increased excretion of these microproteins is a fairly early predictor of the progression of cardiovascular disease [24]. These authors in their dissertations also demonstrated the indisputable fact that in patients with $\mathrm{EH}$ as the concentration of homocysteine increases in the blood (an independent risk factor for cardiovascular disease and increased mortality), significantly increased urinary excretion of B2-MG and N-acetylbeta-D-glucosaminidase (markers of tubular dysfunction). At the time, study data showed that patients with mild hypertension have an increased level of excretion of N-acetyl-beta-Dglucosaminidase, and this enzyme was statistically significantly related to the age of patients, 
sodium excretion and blood glucose, but the interdependence of N-acetyl-beta-Dglucosaminidase and SBP levels was statistically insignificant [25].

It should be noted from the analysis of the data presented in the literature, that the increased excretion of B2-MG may be due to a number of mechanisms, including: excessive filtration through the affected by hypertension glomerular filter B2-MG, exceeding the threshold of reabsorption of the proximal tubular epithelium; dysfunction of the tubular apparatus of the kidneys, due to both generalized changes in intrarenal hemodynamics and impaired blood flow in the paratubular capillaries due to changes in the tone of the efferent arterioles (pathological effect of angiotensin II); nephrotoxic effect of "filtered" albumin on tubulointerstitial tissue with subsequent possible formation of fibrosis and the development of proteinuric remodeling of the interstitium [26]. The above mechanisms form the pathogenetic preconditions for the development of tubular dysfunction and subsequent interstitial sclerosis, which, together with damage and increased permeability of the glomerular filter of nephrons, determines the damage to the kidneys as a target organ in $\mathrm{EH}$ in the formation of hypertensive nephropathy.

Thus, summarizing the above data, it can be noted: the study showed that the method of determining the excretion of B2-MG as a marker of hypertensive nephropathy can be used for this purpose as an effective method with high sensitivity and specificity. Determination of B2-MGU, together with the assessment of urinary albumin excretion, allows to comprehensively characterize not only the severity of permeability of the glomerular filter, but also the dysfunction of the tubular apparatus, which will generally assess the degree of involvement of the kidneys in the early preclinical stage. The high frequency and severity of hyper-B2-MGU in EH, which increased as the disease progressed, the pathogenetic relationship of increased B2-MGU excretion with impaired renal hemodynamics, indicators of daily blood pressure monitoring, the degree of left ventricular myocardial hypertrophy and reduced its pumping function, give grounds to consider it possible additional important clinical and prognostic factor in the development of complications in EH. Studying the effect of different groups of antihypertensive drugs on the dynamics of B2-MGU in patients with EH will assess the organoprotective effect of therapy, and monitoring the excretion of B2-MG in the urine during treatment can be used as a criterion for treatment, which is a promising goal research. 


\section{Conclusions:}

1. In patients with $\mathrm{EH}$ is forming hypertensive nephropathy, which is characterized by statistically significant excessive excretion of B2-MG in the urine compared with almost healthy individuals.

2. The B2-MGU indicator can be used as an adequate marker of hypertensive nephropathy, as having high sensitivity, specificity, positive and negative prognosis.

3. In patients with increased excretion of B2-MG violations of intrarenal hemodynamics were registered significantly more often, violations of functional renal reserve, violations of vascular resistance of renal vessels, in a significantly higher percentage there were pathological changes in circadian rhythm of $\mathrm{BP}$ and renal filtration function, the prevalence of concentric and eccentric hypertrophy, proteinuria, which indicates an important clinical, diagnostic and prognostic value of hyper-B2-MGU.

Prospects for further research. A promising goal of future studies is to assess the effect of combination therapy with inhibitors of the renin-angiotensin system on the state of intrarenal hemodynamics and the functional state of the kidneys.

Conflicts of interest. Neither author has actual or potential conflicts of interest.

\section{References}

1. $\quad \mathrm{Ku}$ E, Lee BJ, Wei J, Weir MR. Hypertension in CKD: Core Curriculum 2019. Am J Kidney Dis. 2019 Jul;74(1):120-131. doi: 10.1053/j.ajkd.2018.12.044. Epub 2019 Mar 19. PMID: 30898362.

2. Freedman BI, Cohen AH. Hypertension-attributed nephropathy: what's in a name? Nat Rev Nephrol. 2016 Jan;12(1):27-36. doi: 10.1038/nrneph.2015.172. Epub 2015 Nov 10. PMID: 26553514.

3. Li XC, Zhang J, Zhuo JL. The vasoprotective axes of the renin-angiotensin system: Physiological relevance and therapeutic implications in cardiovascular, hypertensive and kidney diseases. Pharmacol Res. 2017 Nov;125(Pt A):21-38. doi: 10.1016/j.phrs.2017.06.005. Epub 2017 Jun 12. PMID: 28619367; PMCID: PMC5607101.

4. Girndt M. Diagnostik und Therapie der chronischen Nierenerkrankung [Diagnosis and treatment of chronic kidney disease]. Internist (Berl). 2017 Mar;58(3):243256. German. doi: 10.1007/s00108-017-0195-2. PMID: 28194476.

5. Weisrock F, Fritschka M, Beckmann S, Litmeier S, Wagner J, Tahirovic E, Radenovic S, Zelenak C, Hashemi D, Busjahn A, Krahn T, Pieske B, Dinh W, Düngen HD. Reliability of peripheral arterial tonometry in patients with heart failure, diabetic nephropathy 
and arterial hypertension. Vasc Med. 2017 Aug;22(4):292-300. doi: 10.1177/1358863X17706752. Epub 2017 May 30. PMID: 28555533.

6. Ruiz-Hurtado G, Ruilope LM. Microvascular injury and the kidney in hypertension. Hipertens Riesgo Vasc. 2018 Jan-Mar;35(1):24-29. doi: 10.1016/j.hipert.2017.03.002. Epub 2017 Apr 18. PMID: 28431922.

7. Sievers LK, Eckardt KU. Molecular Mechanisms of Kidney Injury and Repair in Arterial Hypertension. Int J Mol Sci. 2019 Apr 30;20(9):2138. doi: 10.3390/ijms20092138. PMID: 31052201; PMCID: PMC6539752.

8. Boddi M. Renal Ultrasound (and Doppler Sonography) in Hypertension: An Update. Adv Exp Med Biol. 2017;956:191-208. doi: 10.1007/5584_2016_170. PMID: 27966109.

9. Wang Y, Wang C, Zhang X, Gu HF, Wu L. Common Drugs for Stabilization of Renal Function in the Progression of Diabetic Nephropathy and Their Relations with Hypertension Therapy. Curr Diabetes Rev. 2018;14(2):149-161. doi: 10.2174/1573399813666170214112115. PMID: 28201968.

10. Tirapani LDS, Fernandes NMDS. A narrative review of the impacts of income, education, and ethnicity on arterial hypertension, diabetes mellitus, and chronic kidney disease in the world. Saudi J Kidney Dis Transpl. 2019 Sep-Oct;30(5):1084-1096. doi: 10.4103/1319-2442.270264. PMID: 31696847.

11. Yang C, Wang H, Zhao X, Matsushita K, Coresh J, Zhang L, Zhao MH. CKD in China: Evolving Spectrum and Public Health Implications. Am J Kidney Dis. 2020 Aug;76(2):258-264. doi: 10.1053/j.ajkd.2019.05.032. Epub 2019 Sep 3. PMID: 31492486.

12. Sternlicht H, Bakris GL. The Kidney in Hypertension. Med Clin North Am. 2017 Jan;101(1):207-217. doi: 10.1016/j.mcna.2016.08.001. PMID: 27884230.

13. Robinson TW, Freedman BI. The Impact of APOL1 on Chronic Kidney Disease and Hypertension. Adv Chronic Kidney Dis. 2019 Mar;26(2):131-136. doi: 10.1053/j.ackd.2019.01.003. PMID: 31023447; PMCID: PMC6601639.

14. Culver S, Li C, Siragy HM. Intrarenal Angiotensin-Converting Enzyme: the Old and the New. Curr Hypertens Rep. 2017 Sep 19;19(10):80. doi: 10.1007/s11906-0170778-2. PMID: 28929450; PMCID: PMC5913745.

15. Kotsis V. Treatment of Hypertension Induced Target Organ Damage. Curr Pharm Des. 2018;24(37):4377. doi: 10.2174/138161282437190227103636. PMID: 30885112.

16. Kim CS, Choi HS, Bae EH, Kim SW, Ma SK. Optimal blood pressure target and measurement in patients with chronic kidney disease. Korean J Intern Med. 2019 
Nov;34(6):1181-1187. doi: 10.3904/kjim.2019.164. Epub 2019 Jun 17. PMID: 31189302; PMCID: PMC6823567.

17. Quimby JM. Update on Medical Management of Clinical Manifestations of Chronic Kidney Disease. Vet Clin North Am Small Anim Pract. 2016 Nov;46(6):1163-81. doi: 10.1016/j.cvsm.2016.06.004. PMID: 27593576.

18. Emrich IE, Böhm M, Mahfoud F. The 2018 ESC/ESH Guidelines for the management of arterial hypertension: A German point of view. Eur Heart J. 2019 Jun 14;40(23):1830-1831. doi: 10.1093/eurheartj/ehz381. PMID: 31199493.

19. Mukoyama M, Adachi M. [Kidney and hypertension]. Nihon Jinzo Gakkai Shi. 2016;58(1):26-32. Japanese. PMID: 26950978.

20. Costache II, Costea CF, Fotea V, Rusu VL, Aursulesei V, Al Namat R, Costache DA, Dumitrescu N, Buzdugă CM, Dumitrescu GF, Sava A, Bogdănici CM. Morphological and functional renovascular changes as cause of resistant arterial hypertension - case report and literature review. Rom J Morphol Embryol. 2018;59(1):323-328. PMID: 29940645.

21. Fouad M, Fathy H, Zidan A. Serum uric acid and its association with hypertension, early nephropathy and chronic kidney disease in type 2 diabetic patients. J Bras Nefrol. 2016 Dec;38(4):403-410. Portuguese, English. doi: 10.5935/0101-2800.20160065. PMID: 28001185.

22. Kaltsatou A. Management of Blood Pressure and Heart Rate in Chronic Kidney Disease. Curr Pharm Des. 2017;23(31):4603-4608. doi: 10.2174/1381612823666170829164305. PMID: 28875847.

23. Di Nicolò P. The dark side of the kidney in cardio-renal syndrome: renal venous hypertension and congestive kidney failure. Heart Fail Rev. 2018 Mar;23(2):291-302. doi: 10.1007/s10741-018-9673-4. PMID: 29383636.

24. Isaacs $\mathrm{AN}$, Vincent A. Antihypertensive therapy for the prevention of nephropathy in diabetic hypertensive patients. J Clin Pharm Ther. 2016 Apr;41(2):111-5. doi: 10.1111/jcpt.12361. Epub 2016 Feb 6. PMID: 26850093.

25. Andrikou I, Tsioufis C, Konstantinidis D, Kasiakogias A, Dimitriadis K, Leontsinis I, Andrikou E, Sanidas E, Kallikazaros I, Tousoulis D. Renal resistive index in hypertensive patients. J Clin Hypertens (Greenwich). 2018 Dec;20(12):1739-1744. doi: 10.1111/jch.13410. Epub 2018 Oct 25. PMID: 30362245. 
26. Dojki FK, Bakris GL. Blood Pressure Control and Cardiovascular/Renal Outcomes. Endocrinol Metab Clin North Am. 2018 Mar;47(1):175-184. doi: 10.1016/j.ecl.2017.10.008. PMID: 29407050. 